\title{
Strength properties of a Drucker-Prager porous medium reinforced by rigid particles
}

\author{
$\underline{\text { Zheng He }}^{1, *}$, Luc Dormieux ${ }^{1}$, Kondo Djimedo ${ }^{2}$ \\ ${ }^{1}$ E.N.P.C, Institut Navier, 77455 Marne-la-Vallee, France \\ ${ }^{2}$ U.M.P.C, Institut d'Alembert, 75252 PARIS CEDEX 05, France \\ *Corresponding author: hez@lmsgc.enpc.fr
}

\begin{abstract}
In the present study, we investigate the strength properties of ductile porous materials reinforced by rigid particles. The microporous medium is constituted of a Drucker-Prager solid phase containing spherical voids; its behaviour is described by means of an elliptic criterion (issued from a modified secant moduli approach) whose corresponding support function is determined. The later is then implemented in a limit analysis approach in which a careful attention is paid for the contribution of the inclusion matrix-interface. This delivers parametric equations of the effective strength properties of the porous material reinforced by rigid particles. The predictions are compared to available results obtained by means of variationnal homogenization methods successively applied for micro-to-meso and then for meso-to-macro scales transitions. Moreover, we discuss in detail the predictions of the material strength under isotropic mechanical loadings. To this end, additional static solutions are derived and compared to the kinematics limit analysis ones. Finally, we derive an approximate closed-form expression of the macroscopic strength which proves to be very accurate.
\end{abstract}

Keywords Porous; Strength; Drucker-Prager; inclusion-matrix, interfaces.

\section{Introduction}

Being hard clayey rocks, COx Argillite is a porous clay matrix in which quartz or silica inclusions are embedded. In the present study, we mainly aim to derive new closed-form results for the strength of the COx argillite, under the assumption that the solid phase of the clay is a Drucker-Prager perfectly plastic material. Therefore, the behaviour of the microporous clay is described by means of an elliptic criterion [2] whose corresponding support function is determined in this paper. Then by using this support function, we explore an alternative approach which can be viewed as an extension of the original Gurson model. Instead of a spherical cavity surrounded by a matrix, the proposed 'rigid core sphere model' consists of a rigid spherical core surrounded by the homogeneous porous material. The failure criterion of this 'rigid core sphere model' is derived in the framework of the cinematic approach of limit analysis (LA). It is worth noting that from the LA point of view the failure mechanism can include a strain concentration at the core-matrix interface which can be described mathematically. ([1-3],[6],[9],[11],[12]). Notations: 1 and $\mathbb{I}$ are the second and fourth order identity tensors. $\mathbb{J}=(1 / 3) \mathbf{1} \otimes \mathbf{1}, \mathbb{K}=\mathbb{I}-\mathbb{J}$ are respectively the spherical and deviatoric projectors of isotropic fourth order symmetric tensor.

\section{The micro-to-meso transition: support function of porous matrix}

The first homogenization step approach starts at the microscopic scale. At this scale, the porous clay matrix is described as a heterogeneous material being made up of a Drucker-Prager perfectly plastic solid in which pores are embedded. Let $\tilde{\sigma}_{d}=\tilde{\sigma}-\tilde{\sigma}_{m} 1$ denote the deviatoric part of the stress tensor $\tilde{\sigma}$ at the microscopic scale. The scalar deviatoric stresses $\tilde{\sigma}_{m}$ are defined as $\tilde{\sigma}_{d}=\sqrt{\tilde{\sigma}: \mathbb{K}: \tilde{\sigma}}$ and $\tilde{\sigma}_{m}=(1: \mathbb{J}: \tilde{\sigma}) / 3$ and the Drucker-Prager criterion reads: 


$$
\tilde{\sigma}_{d}+T\left(\tilde{\sigma}_{m}-h\right) \leq 0 .
$$

The parameters $T$ and $h$ respectively characterize the friction coefficient and the tensile strength of the solid phase of the clay matrix.

The result of the first homogenization step is the derivation of the strength properties of the porous clay matrix at the mesoscopic scale where it is described as a homogeneous material. These properties were estimated successfully in [6] by means of the modified secant method. Now $\sigma_{d}=\sigma-\sigma_{m} 1$ denotes the deviatoric part of the stress tensor $\sigma$ at the mesoscopic scale and we introduce $\sigma_{d}=\sqrt{\sigma: \mathbb{K}: \sigma}$ and $\sigma_{m}=(\mathbf{1}: \mathbb{J}: \sigma) / 3$. In the situation of associated plasticity, the domain of admissible stress states is an ellipse in the $\left(\sigma_{\mathrm{m}}, \sigma_{\mathrm{d}}\right)$-plane:

$$
F^{\text {meso }}(\sigma, f, T)=\frac{1+2 f / 3}{T^{2}} \sigma_{d}^{2}+\left(\frac{3 f}{2 T^{2}}-1\right) \sigma_{m}^{2}+2(1-f) h \sigma_{m}-(1-f)^{2} h^{2} \leq 0 .
$$

Note that $0<T \leq \sqrt{3 f / 2}$ (see [6]), $f$ is the porosity. At the mesoscopic scale, the clay matrix is described by the elliptic criterion (2). In the framework of limit analysis theory (see e.g. [10]), a dual characterization of the strength criterion $F(\sigma) \leq 0$ is the support function $\pi_{F}(\mathbf{d})=\sup (\sigma: \mathbf{d}, F(\sigma) \leq 0\}$ of the convex set of admissible stress states. The support function $\pi(\mathbf{d})$ associated with (2) of the porous matrix finally takes the form

$$
\begin{gathered}
\pi_{F}(\mathbf{d})=\sigma_{0} d_{E Q}-\lambda d_{v} \quad \text { with } \quad d_{E Q}=\sqrt{\frac{2}{3} \mathbf{d}: H: \mathbf{d} .} \\
\sigma_{0}=(1-f) h \sqrt{\frac{3}{2} \sqrt{\frac{3 f}{3 f-2 T^{2}} \frac{T^{2}}{1+2 f / 3}}} \quad ; \quad \lambda=(1-f) h \frac{2 T^{2}}{3 f-2 T^{2}} .
\end{gathered}
$$

where $\mathbb{H}=\frac{1}{\alpha} \mathbb{J}+\mathbb{K}$ is a fourth order tensor, with

$$
\alpha=\frac{3 f / 2-T^{2}}{3+2 f} .
$$

\section{Overall dissipation at the mesoscopic scale}

We now focus on the transition from the mesoscopic scale to the macroscopic scale which constitutes the second homogenization step and is the main subject of the present paper. We seek the macroscopic criterion by means of a Gurson-type approach. As already stated, the microstructure at the mesoscopic scale is described by a composite sphere $\Omega$ with a rigid core surrounded by the homogenized clay resulting from the micro-to-meso transition. The external (resp. internal) radius is denoted by $r_{e}$ (resp. $\left.r_{i}\right)$. The shell $\Omega_{m}\left(r_{i} \leq r \leq r_{e}\right)$ around the core represents the clay. The volume fraction $\rho=\left(r_{i} / r_{e}\right)^{3}$ of the core in the composite sphere is equal to the volume fraction of the rigid inclusions in a representative volume element of argillite.

\subsection{Velocity field at the mesoscopic scale}

For geomaterials, we define here a family of cinematically admissible (k.a.) velocity fields with $\mathbf{D}$, depending on one compressible parameter $A$ :

$$
r \geq r_{i}: \quad \underline{v}_{A}=A \underline{x}+\left(D_{m}-A\right) \frac{r_{e}^{3}}{r^{2}} \underline{e}_{r}+\mathbf{D}_{d} \cdot \underline{x} .
$$

The strain rate in the clay $\left(r_{i} \leq r \leq r_{e}\right)$ can be determined from (6): 


$$
\mathbf{d}_{A}=A 1+\mathbf{D}_{d}+\left(D_{m}-A\right) \frac{r_{e}^{3}}{r^{3}}\left(1-3 \underline{e}_{r} \otimes \underline{e}_{r}\right)
$$

From (7), with $D_{d}^{2}=\mathbf{D}_{\mathbf{d}}: \mathbf{D}_{\mathbf{d}}$, the expression of $d_{E Q}$ in (3) can be written as

$$
d_{E Q}^{2}=\frac{2 A^{2}}{\alpha}+4\left(D_{m}-A\right)^{2} \frac{r_{e}^{6}}{r^{6}}+\frac{2 D_{d}^{2}}{3}+\frac{4\left(D_{m}-A\right)}{3} \frac{r_{e}^{3}}{r^{3}} \mathbf{D}_{d}:\left(1-3 \underline{e}_{r} \otimes \underline{e}_{r}\right)
$$

The velocity is $\underline{v}_{O}=0$ in the rigid core. Note that the condition $\underline{v}_{A}=0$ on the boundary $r=r_{i}$ cannot be fulfilled by the velocity field defined in (6). This implies that the dissipation associated with a discontinuity of velocity must be considered at the boundary $I\left(r=r_{i}\right)$ (section 3.4).

\subsection{Macroscopic support function}

Defining the macroscopic strength domain $G^{\text {hom }}$ as the set of admissible macroscopic stress states $\Sigma$, the macroscopic support function reads $\Pi^{\text {hom }}(\mathbf{D})=\sup \left(\Sigma: \mathbf{D}, \Sigma \in G^{\text {hom }}\right)$. Considering the set $K$ of k.a. velocity fields with $\mathbf{D}, \Pi^{\text {hom }}(\mathbf{D})$ is characterized as [5]:

$$
\Pi^{\text {hom }}(\mathbf{D})=\frac{1}{|\Omega|} \inf _{\mathbf{v} \in K}\left(\int_{\Omega_{m}} \pi_{F}\left(\mathbf{d}_{A}\right) d V+\int_{I} \pi\left(\underline{v}_{A}\right) d S\right) .
$$

where $|\Omega|=4 \pi r_{e}^{3} / 3$. In the surface integral, $\underline{v}$ denotes the velocity discontinuity at the core boundary $I$ and $\pi(\underline{v})$ represents the associated surface density of dissipation. In the line of reasoning of Gurson approach, $\Pi^{\text {hom }}(\mathbf{D})$ is approximated by the minimal dissipation obtained among the velocity fields $\underline{v}_{A}$ defined in (6):

$$
\Pi^{\text {hom }}(\mathbf{D})=\frac{1}{|\Omega|} \inf _{A \in R}\left(\int_{\Omega_{m}} \pi_{F}\left(\mathbf{d}_{A}\right) d V+\int_{I} \pi\left(\underline{v}_{A}\right) d S\right) .
$$

For further use, let us introduce the following notation:

$$
\frac{1}{|\Omega|} \int_{\Omega_{m}} \pi_{F}\left(\mathbf{d}_{A}\right) d V=\tilde{\Pi}^{m}(\mathbf{D}, A) \quad ; \quad \frac{1}{|\Omega|} \int_{I} \pi\left(\underline{v}_{A}\right) d S=\tilde{\Pi}^{I}(\mathbf{D}, A) .
$$

Accordingly:

$$
\begin{gathered}
\Pi^{h o m}(\mathbf{D})=\inf _{A \in R} \tilde{\Pi}(\mathbf{D}, A) . \\
\tilde{\Pi}(\mathbf{D}, A)=\tilde{\Pi}^{m}(\mathbf{D}, A)+\tilde{\Pi}^{I}(\mathbf{D}, A) .
\end{gathered}
$$

Once $\Pi^{\text {hom }}(\mathbf{D})$ is determined, the macroscopic admissible stress states on the boundary $\partial G^{\text {hom }}$ are derived according to:

$$
\Sigma=\frac{\partial \Pi^{h o m}}{\partial \mathbf{D}}(\mathbf{D})
$$

The stress state of (14) lies on the boundary of $G^{\text {hom }}$ at the location where the normal is parallel to D. The overall dissipation of (12) proves to read in the following form: 


$$
\tilde{\Pi}(\mathbf{D}, A)=\sigma_{0}\left[\operatorname{Narcsinh}\left(\frac{u N}{M}\right)-\frac{\sqrt{M^{2}+u^{2} N^{2}}}{u}\right]_{1}^{\frac{1}{\rho}}+\sigma_{0} \sqrt{\frac{2}{3}} Y-3 \lambda D_{m} .
$$

with the notations introduced in (19), (27) and (28). For the sake of completeness, sections 3.3 and 3.4 respectively determine the contribution (18) of the shell $\Omega_{m}$ (volume integral in (10)) and the contribution (26) of the interface $I$ (surface integral in (10)) which has led to (15). Section 4 will consider the minimization w.r.t. parameter $A$.

\subsection{Contribution of the shell to dissipation}

For a given value of parameter $A$, the contribution of the matrix to the macroscopic dissipation reads

$$
\tilde{\Pi}^{m}(\mathbf{D}, A)=\frac{1}{|\Omega|} \int_{\Omega_{m}}\left(\sigma_{0} d_{E Q}-\lambda d_{v}\right) d V
$$

In order to obtain an analytical expression of $\tilde{\Pi}^{m}(\mathbf{D}, A)$, the approximation introduced in [4] is applied. Let $S(r)$ denote the sphere of radius $r$. As a consequence of the Cauchy-Schwarz inequality, it is readily seen that

$$
\int_{S(r)} d_{E Q} d S \leq \sqrt{4 \pi r^{2}} \sqrt{\int_{S(r)} d_{E Q}^{2} d S}
$$

We observe that the average $\left\langle\mathbf{1}-3 \underline{e}_{r} \otimes \underline{e}_{r}\right\rangle_{S(r)}$ of $\mathbf{1}-3 \underline{e}_{r} \otimes \underline{e}_{r}$ over the orientations of $\underline{e}_{r}$ on the sphere $S(r)$ is equal to 0 . Then, using (8) and (17) in $(16), \tilde{\Pi}^{m}(\mathbf{D}, A)$ reads

$$
\begin{aligned}
\tilde{\Pi}^{m}(\mathbf{D}, A) & =\frac{4 \pi \sigma_{0}}{\Omega} \int_{r_{i}}^{r_{e}} r^{2} \sqrt{\frac{2 A^{2}}{\alpha}+4\left(D_{m}-A\right)^{2} \frac{r_{e}^{6}}{r^{6}}+\frac{2 D_{d}^{2}}{3}} d r-\frac{\lambda}{\Omega} \int_{\Omega^{m}} d_{v} d V \\
& =\sigma_{0}\left[\operatorname{Narcsinh}\left(\frac{u N}{M}\right)-\frac{\sqrt{M^{2}+u^{2} N^{2}}}{u}\right]_{1}^{\frac{1}{\rho}}-3(1-\rho) \lambda A
\end{aligned}
$$

with

$$
M^{2}=\frac{2 A^{2}}{\alpha}+\frac{2 D_{d}^{2}}{3}, \quad N^{2}=4\left(D_{m}-A\right)^{2} .
$$

\subsection{Inclusion-matrix interface}

Unlike the classical Gurson's 'hollow sphere model', the model proposed in this paper substitutes a rigid core for the void in the center of the thick-walled sphere. Therefore, owing to null velocity $\left(\underline{v}_{O}=0\right)$ in the rigid core, a velocity discontinuity tales place at the core boundary: $\underline{v}_{A}=\underline{v}_{A}\left(r_{a} \underline{e}_{r}\right)-\underline{v}_{O}=\underline{v}_{A}\left(r_{a} \underline{e}_{r}\right)$.

\subsubsection{Surface density of dissipation}

The velocity field $\underline{v}_{A}$ being discontinuous across the surface $I$ (rigid core boundary), its gradient and the associated strain rate are to be defined in the sense of the distribution theory: 


$$
\mathbf{d}=\{\mathbf{d}\}+\frac{1}{2}\left(\underline{n} \otimes \underline{v}_{A}+\underline{v}_{A} \otimes \underline{n}\right) \delta_{I} .
$$

where $\{\mathbf{d}\}$ is the standard expression of the strain rate corresponding to its smooth part, $\delta_{I}$ is the Dirac distribution which support is the surface of discontinuity $I$ and $\underline{n}=\underline{e}_{r}$ is the unit normal to this surface. The surface density of dissipation $\pi\left(\underline{v}_{A}\right)$ contributed by the velocity jump $\underline{v}_{A}$ is therefore related to the support function $\pi_{F}$ according to (see e.g. [10]):

$$
\pi\left(\underline{v}_{A}\right)=\pi_{F}\left(\mathbf{d}^{I}\right) \text {. }
$$

where $\mathbf{d}^{I}$ is defined as

$$
\mathbf{d}^{I}=\frac{1}{2}\left(\underline{n} \otimes \underline{v}_{A}+\underline{v}_{A} \otimes \underline{n}\right) .
$$

Recalling (6) and (22), the strain rate $\mathbf{d}^{I}$ associated with the velocity jump can be obtained and written as

$$
\mathbf{d}^{I}=r_{i}\left(\left(\frac{1}{\rho} D_{m}+\left(1-\frac{1}{\rho}\right) A\right) \underline{e}_{r} \otimes \underline{e}_{r}+\frac{1}{2}\left(\left(\mathbf{D}_{d} \cdot \underline{e}_{r}\right) \otimes \underline{e}_{r}+\underline{e}_{r} \otimes\left(\mathbf{D}_{d} \cdot \underline{e}_{r}\right)\right)\right)
$$

Eventually, the surface density of dissipation is derived from the combination of (21) and (3)

$$
\pi\left(\underline{v}_{A}\right)=\sigma_{0} d_{E Q}^{I}-\lambda d_{v}^{I} \quad \text { with } \quad d_{E Q}^{I}=\sqrt{\frac{2}{3} \mathbf{d}^{I}: H: \mathbf{d}^{I}} \quad ; \quad d_{v}^{I}=\operatorname{tr} \mathbf{d}^{I} .
$$

\subsubsection{Contribution of the interface to dissipation}

Recalling (16), the macroscopic dissipation related to the part of inclusion-matrix interface depending also on the scalar $A$ can be written as

$$
\tilde{\Pi}^{I}(\mathbf{D}, A)=\frac{1}{\Omega} \int_{r=r_{i}}\left(\sigma_{0} d_{E Q}^{I}-\lambda d_{v}^{I}\right) d S
$$

Again, the integration of $d_{e q}^{I}$ is approximated by the upper bound (17) which yields

$$
\tilde{\Pi}^{I}(\mathbf{D}, A)=\sigma_{0} \sqrt{\frac{2}{3}} Y-\lambda X \quad ; \quad Y=\frac{\sqrt{4 \pi r_{i}^{2}}}{\Omega} \sqrt{\int_{r=r_{i}} \mathbf{d}^{I}: H: \mathbf{d}^{I} d S} .
$$

Using (23), $Y$ takes the form

with

$$
Y=\sqrt{\frac{P^{2}+Q^{2}}{15 \alpha}}
$$

$$
Q^{2}=\left(\frac{51}{2} \alpha+6\right) \rho^{2} D_{d}^{2}, \quad P^{2}=45(1+2 \alpha)\left[D_{m}-A(1-\rho)\right]^{2}
$$

In turn, observing that the average $\left\langle\underline{e}_{r} \cdot \mathbf{D}_{d} \cdot \underline{e}_{r}\right\rangle_{r=r_{i}}$ of $\left\langle\underline{e}_{r} \cdot \mathbf{D}_{d} \cdot \underline{e}_{r}\right\rangle_{r=r_{i}}$ over the orientations of $\underline{e}_{r}$ on the sphere $I$ is equal to 0 , it is readily seen that

$$
X=\frac{1}{\Omega} \int_{S\left(r_{a}\right)} d_{v}^{I} d S=3\left(D_{m}-A(1-\rho)\right) .
$$




\section{Macroscopic criterion}

The macroscopic support function can be determined by minimizing the sum $\tilde{\Pi}(\mathbf{D}, A)=\tilde{\Pi}^{m}(\mathbf{D}, A)+\tilde{\Pi}^{I}(\mathbf{D}, A)$ with respect to the parameter A. Accordingly, the boundary of $G^{\text {hom }}$ is determined according to (14)[7]:

$$
\Sigma=\frac{\partial \tilde{\Pi}(\mathbf{D}, A)}{\partial \mathbf{D}}, \quad \text { with } \quad \frac{\partial \tilde{\Pi}(\mathbf{D}, A)}{\partial A}=0 .
$$

\section{Comparison with the result obtained by a variational approach}

Predictions according to (30) of the macroscopic criterion derived in the framework of the cinematic approach of limit analysis are now compared with the result obtained by the variational approach [11]. For the derivation of their criterion, these authors consider a variational approach in the two homogenization steps. Their criterion reads:

$$
F^{\mathrm{hom}}(\Sigma, f, T)=\Theta \Sigma_{d}^{2}+\left(\frac{3 f}{2 T^{2}}-1\right) \Sigma_{m}^{2}+2(1-f) h \Sigma_{m}-\frac{3+2 f+3 f \rho}{3+2 f}(1-f)^{2} h^{2}=0
$$

with

$$
\Theta=\frac{\frac{1+2 f / 3}{T^{2}}+\frac{2}{3} \rho\left(\frac{3 f}{2 T^{2}}-1\right)}{\frac{4 T^{2}-12 f-9}{6 T^{2}-13 f-6} \rho+1} .
$$

Applying the parameters $f=0.25$ and $T=0.525$, the comparison between the results predicted by the two different methods is shown in Fig.1 As it can be seen in Fig.1, the analytical estimate (31) obtained by the variational approach and the prediction from (30) based on the 'rigid core sphere model' show a very good agreement for purely isotropic stress states, both in traction and compression. It is noteworthy that the strength under purely isotropic stress seems surprisingly almost unaffected by the volume fraction $\rho$ of the rigid core. Although the shapes of the yield surfaces predicted by the two methods are similar, the strength predicted by limit analysis always overestimates that predicted by the variational method. In particular, as far as the strength under pure shear loading is concerned, the difference becomes very important when the volume fraction of the rigid core $\rho$ is larger. In order to gain a deeper understanding of the effect of the parameter $\rho$, the isotropic strength will now be compared with the exact solution predicted by the so-called static approach (sections 6). On the other hand, we note that the strength predicted by limit analysis (upper bounds) overestimates that predicted by the variational method under shear loading.(We focus on the strength proprieties under isotropic loading. The strength under shear loading has not been discussed in the present paper.)

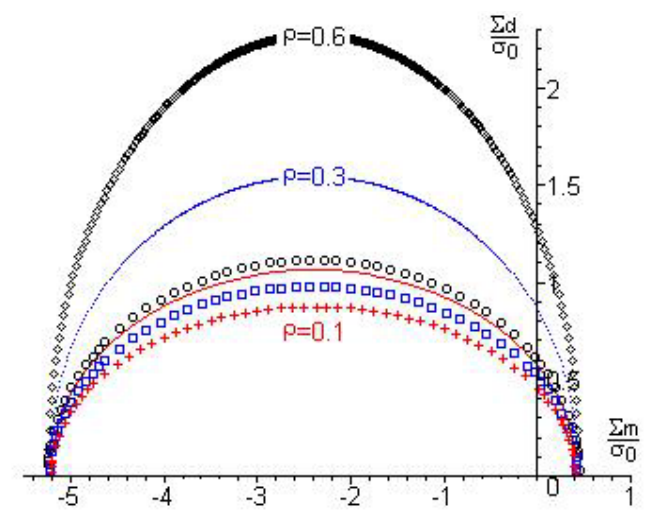


Figure 1. Comparison between the results predicted by limit analysis and the variational approach for different volume fractions of the rigid inclusion

-'line': limit analysis with $\rho=0.1, \quad$-'cross': variational approach with $\rho=0.1$,

-'point': limit analysis with $\rho=0.3, \quad$-'box': variational approach with $\rho=0.3$,

-'diamond': limit analysis with $\rho=0.6$, -'circle': variational approach with $\rho=0.6$

\section{Strength under isotropic loading}

\subsection{Static approach of the limit analysis problem}

The theory of limit analysis teaches that a cinematic approach like the Gurson one provides an upper bound of the true strength. In order to check the accuracy of a cinematic estimate, it is therefore highly desirable to derive a static approach which in turn will deliver a lower bound of the true strength. We therefore seek the stress field solution to an isotropic loading (traction or compression) in the framework of the 'rigid core sphere model'. The macroscopic stress state is of the form $\Sigma_{m} 1$. Accordingly the external boundary $\left(r=r_{e}\right)$ is subjected to a radial surface force $\sigma \cdot \underline{n}=\Sigma_{m} \underline{n}$. We implement the so-called static approach of limit analysis. It consists in deriving a mesoscopic stress field $\sigma$ which must be statically admissible with these boundary conditions and meet the criterion $F^{\text {meso }}(\sigma)=0$ (see (2)). Owing to the spherical symmetry, this statically admissible stress field can be sought in the form (spherical coordinates):

$$
\sigma=\sigma_{r r}(r) \underline{e}_{r} \otimes \underline{e}_{r}+\sigma_{\theta \theta}(r)\left(\underline{e}_{\theta} \otimes \underline{e}_{\theta}+\underline{e}_{\varphi} \otimes \underline{e}_{\varphi}\right) \text {. }
$$

The boundary condition on the surface $r=r_{e}$ reads

$$
\sigma_{r r}\left(r_{e}\right)=\Sigma_{m} \text {. }
$$

The momentum balance equation $\operatorname{div} \sigma=0$ reduces to

$$
\frac{d \sigma_{r r}}{d r}=2 \frac{\sigma_{\theta \theta}-\sigma_{r r}}{r} \text {. }
$$

With the notation $X=\sigma_{\theta \theta}-\sigma_{r r}$ (note that $X^{2}=\frac{3}{2} \sigma_{d}^{2}$ ), it is found that $\sigma_{m}=\sigma_{r r}-\frac{2}{3} X$, so that the criterion (2) yields

$$
\frac{2}{3} \frac{\left(1+\frac{2}{3} f\right) X^{2}}{T^{2}}+\frac{3 f-2 T^{2}}{2 T^{2}}\left(\sigma_{r r}+\frac{2}{3} X\right)^{2}+2(1-f) h\left(\sigma_{r r}+\frac{2}{3} X\right)-(1-f)^{2} h^{2}=0 .
$$

The values of $X$ solutions of (36) read:

$$
\begin{gathered}
X=\frac{3}{2} \frac{\left(3 f-2 T^{2}\right) \sigma_{r r}-2 h T^{2}(f-1) \pm \sqrt{\Delta}}{2 T^{2}-5 f-3}, \text { with . } \\
\Delta=(2 f+3)\left(2 T^{2}-3 f\right) \sigma_{r r}^{2}+4 h T^{2}(2 f+3)(f-1) \sigma_{r r}+2 h^{2} T^{2}(5 f+3)(f-1)^{2} .
\end{gathered}
$$

Recalling (35), an ordinary differential equation with respect to $\sigma_{r r}$ is obtained in the form:

$$
X\left(\sigma_{r r}\right)=\frac{r}{2} \frac{d \sigma_{r r}}{d r} .
$$

Introducing (37) into (39) and integrating over the interval $\left[r_{i}, r_{e}\right]$, one obtains 


$$
\frac{1}{3} \ln (\rho)=\int_{c}^{\Sigma_{m}} \frac{1}{3} \frac{2 T^{2}-5 f-3}{\left(2 T^{2}-3 f\right) \sigma_{r r}+2 h T^{2}(f-1)+\varepsilon \sqrt{\Delta}} d \sigma_{r r} .
$$

where the notation $c=\sigma_{r r}\left(r_{a}\right)$ and the boundary condition (34) at $r=r_{e}$ have been used. Note that no boundary condition is available at $r=r_{i}$. The physical meaning of (40) is the following: Whenever there exists a constant $c$ such that (40) is fulfilled (with $\varepsilon=+1$ or -1 ), then $\Sigma_{m}$ is an admissible loading for the value of $\rho$ at stake. We seek the highest possible value $\Sigma_{m}^{+}>0$ of $\Sigma_{m}$ (isotropic strength in traction) and the lowest one, denoted by $\Sigma_{m}^{-}<0$ (isotropic strength on compression). For the simplification of the following discussion, the denominator in the integral of (40) is denoted by $D_{\varepsilon}$ :

$$
D_{\varepsilon}=\left(2 T^{2}-3 f\right) \sigma_{r r}+2 h T^{2}(f-1)+\varepsilon \sqrt{\Delta}
$$

In order for this integral to be defined, two mathematical conditions are to be met, namely $\Delta \geq 0$ and $D_{\varepsilon} \neq 0$. This remark leads to introduce the solutions to the equations of $\Delta=0$ and of $D_{\varepsilon}=0$

First, let $\Sigma_{1 m}^{ \pm}$denote the solutions to $\Delta=0$, which read:

$$
\begin{aligned}
& \Sigma_{1 m}^{+}=\frac{\left[2 T(3+2 f)-\sqrt{6 f(3+2 f)\left(5 f-2 T^{2}+3\right)}\right](1-f) h T}{(3+2 f)\left(2 T^{2}-3 f\right)} \\
& \Sigma_{1 m}^{-}=\frac{\left[2 T(3+2 f)+\sqrt{6 f(3+2 f)\left(5 f-2 T^{2}+3\right)}\right](1-f) h T}{(3+2 f)\left(2 T^{2}-3 f\right)} .
\end{aligned}
$$

Secondly, let $\Sigma_{2 m}^{+}$(resp. $\left.\Sigma_{2 m}^{-}\right)$denote the solution to $D_{+}=0 \quad\left(\right.$ resp. $\left.D_{-}=0\right)$ :

$$
\begin{aligned}
& \Sigma_{2 m}^{-}=\frac{(2 T+\sqrt{6} \sqrt{f})(f-1) T h}{3 f-2 T^{2}} . \\
& \Sigma_{2 m}^{+}=\frac{(2 T-\sqrt{6} \sqrt{f})(f-1) T h}{3 f-2 T^{2}} .
\end{aligned}
$$

After some reasoning, the static solution can be finally determined by numerical integration. Then the static solution is compared to the cinematic solution in the following subsection.

\subsection{Comparison between static and cinematic solutions}

The comparison between the static solution and the cinematic solution as functions of the rigid core volume fraction $\rho(f=0.25$ and $T=0.525)$ has been performed. It is found that the two solutions can hardly be differentiated. it can be concluded that they can be regarded as the exact strength of the composite material, within the rigid core model. .

\subsection{Analytical expressions of the strength under isotropic loading}

Due to the complexity of the integrals in (40), these equations can hardly be solved analytically. 
However, we observe that $\Sigma_{m}^{ \pm}$remain in the neighborhood of $\Sigma_{2 m}^{ \pm}$. We therefore propose to approximate the functions $D_{+}$and $D_{-}$by series expansions in the neighborhood of $\Sigma_{2 m}^{+}$and $\Sigma_{2 m}^{-}$. In the case of of series expansion to the second order, The analytical solutions at order 2 read:

$$
\Sigma_{m}^{ \pm}=\eta+\rho \frac{c-\eta}{\kappa(c-\eta)(1-\rho)+1} .
$$

with the following parameters for isotropic compression or traction:

$$
\left\{\begin{array} { c } 
{ \eta = \Sigma _ { 2 m } ^ { - } } \\
{ \kappa = \frac { 1 } { 2 \sqrt { 6 } ( f - 1 ) \sqrt { f } h T } } \\
{ c = \Sigma _ { 1 m } ^ { - } }
\end{array} \text { or } \quad \left\{\begin{array}{c}
\eta=\Sigma_{2 m}^{+} \\
\kappa=\frac{1}{2 \sqrt{6}} \frac{3+2 f}{(1-f) \sqrt{f} h T} \\
c=\Sigma_{1 m}^{+}
\end{array} .\right.\right.
$$

\section{An approximate analytical macroscopic criterion}

We seek an approximation of the criterion by an ellipse in the $\left(\Sigma_{m}, \Sigma_{d}\right)$ plane. The ellipse intersects the $\Sigma_{m}$-axis at the points $\left(\Sigma_{m}^{-}, 0\right)$ and $\left(\Sigma_{m}^{+}, 0\right)$. The center of the ellipse is located at the middle of these points. We still have to determine the ordinate of the center of the ellipse which corresponds to the strength under pure shear $\left(D_{m}=0\right)$.

With the condition $D_{m}=0$, it is readily seen that the value of the parameter $A$ which minimizes $\tilde{\Pi}(\mathbf{D}, A)$ is $A=0$. With $D_{m}=0$ and $A=0$, closed-form expressions of the mean stress and the maximum shear stress can be obtained from (30):

$$
\Sigma_{m, c}=-\lambda \quad ; \quad \Sigma_{d, c}=\sigma_{0}\left[\sqrt{\frac{2}{3}}(1-\rho)+\sqrt{\frac{17 \alpha+4}{15 \alpha}} \rho\right] .
$$

where $\lambda, \sigma_{0}, \alpha$ have been defined in (4) and (5). The subscript $c$ recalls that this point is the center of the ellipse.

Now let us try to approximate the criterion given in parametric form (obtained by means of cinematic approach) found in section 4 by an analytical elliptic criterion. Analytical expressions of the strength have been established at particular stress states, namely under isotropic loading and under pure shear loading (with $\Sigma_{m}=-\lambda$ ). Recalling (45) (46) for the expressions of $\Sigma_{m}^{+}, \Sigma_{m}^{-}$in the case of a second order expansion, together with (47) for the expression of $\Sigma_{d, c}$, the macroscopic criterion can be approached by the following analytical elliptic function:

$$
\left(\frac{\Sigma_{m}-\lambda}{\Sigma_{L} / 2}\right)^{2}+\left(\frac{\Sigma_{d}}{\Sigma_{d, c}}\right)^{2}=1
$$

with $\Sigma_{L}=\Sigma_{m}^{+}-\Sigma_{m}^{-}$and $\lambda=\left(\Sigma_{m}^{+}+\Sigma_{m}^{-}\right) / 2$. Recall that $\Sigma_{m}^{+}, \Sigma_{m}^{-}, \Sigma_{d, c}$ are quantified in (45),(47) by (42),(43),(44); and $\lambda, \sigma_{0}, \alpha$ are given in (4),(5).

According to the comparison between the predictions of the analytical macroscopic criterion, (48), and the parametric criterion predicted by (30). We found that the comparison shows an excellent accuracy of (48). 


\section{Conclusion}

On the basis of a limit analysis approach, we have proposed an extension of available models (devoted to the macroscopic strength of porous media). This extension concerns porous materials with a Drucker- Prager solid phase, reinforced by rigid particles. The proposed model concerns in particular, the Callovo Oxfordian clay as a composite material made up of rigid inclusions embedded in a porous clay matrix. The obtained results has been compared to the estimate of the strength recently derived by [4] on the basis of a variational non linear homogenization approach. A good accuracy of the estimate of the strength under isotropic loadings has been shown by a comparison with the results of a static (stress based) approach of the limit analysis problem. An interesting observation is that the estimates of the isotropic strength in traction or in compression do not depend on the homogenization method (limit analysis, variational method). Furthermore, the isotropic strength proves to be only slightly affected by the rigid core volume fraction. The practical implication is that the isotropic strength properties of the clay matrix and of the Callovo Oxfordian argillite are very close, irrespective of the quartz/calcite content. In contrast, a significant discrepancy between the failure envelopes is observed on the shear strength for large values of the rigid inclusions concentration.

\section{Acknowledgements}

The work presented in this paper was partly funded by ANDRA, the French national Agency for the management of radioactive wastes, which is gratefully acknowledged.

\section{References}

[1] A. Abou-Chakra Guery, F. Cormery, J.-F. Shao, D. Kondo, 2008. A micromechanical model of elasto-plastic and damage behavior of a cohesive geomaterial. Int. J. Solid. Struct., 45 (5), 1406-1429.

[2] Andra, 2005. Referentiel du site meuse-haute marne. Report.

[3] L. Dormieux, D. Kondo, 2010. An extension of gurson model incorporating interface stresses effects. International Journal of Engineering Science, 48(6), 575-581.

[4] A.L. Gurson, 1977. Continuum theory of ductile rupture by void nucleation and growth: Part I-Yield criterion and flow rules for porous ductile media, J. Engrg. Mat. Technol. 99, 2-15.

[5] J.-B. Leblond, G. Perrin, P. Suquet., 1994. Exact results and approximate models for porous viscoplastic solids. International Journal of Plasticity, 10(3):213-235.

[6] S. Maghous, L. Dormieux, J. Barthelemy, 2009. Micromechanical approach to the strength properties of frictional geomaterials. European Journal of Mechanics A/Solids., 28, 179-188.

[7] V. Monchiet, E. Charkaluk, D. Kondo, 2007. An improvement of Gurson-type models of porous materials by using Eshelby-like trial velocity fields. Comptes Rendus Mecanique., 335(1), Pages 32-41

[8] P.Ponte Castaneda, 1991. The effective mechanical properties of nonlinear isotropic composites. J. Mech. Phys. Solids, 39, 45-71.

[9] J-C. Robinet, P. Sardini, D. Coelho, J-C. Parneix, D. Pret, S. Sammartino, E. Boller and S. Altmann., 2012. Effects of mineral distribution at mesoscopic scale on solute diffusion in a clay-rich rock: Example of the Callovo-Oxfordian mudstone (Bure, France). Water Resources Research, 48, W05554, doi:10.1029/2011WR011352.

[10] J. Salencon, 1990. An introduction to the yield theory and its applications to soil mechanics. European Journal of Mechanics A/Solids, 9(5),477-500.

[11] W.Q. Shen, L. Dormieux, D. Kondo, J.F. Shao, 2013. A closed-form three scale model for ductile rocks with a plastically compressible porous matrix, Mechanics of Materials, 59 73--86

[12] W.Q. Shen, J.F. Shao, D. Kondo, B. Gatmiri., 2012. A micro-macro model for clayey rocks with a plastic compressible porous matrix, International Journal of Plasticity, 36, 64-85 